University of Nebraska - Lincoln

DigitalCommons@University of Nebraska - Lincoln

2010

\title{
An examination of the construct validity of posttraumatic stress disorder with veterans using a revised criterion set
}

Anouk L. Grubaugh

Veterans Affairs Medical Center

Mary E. Long

Michael E. Debakey Veterans Affairs Medical Center and Baylor College of Medicine

Jon D. Elhai

University of Toledo

B. Christopher Frueh

The Menninger Clinic and Baylor College of Medicine

Kathryn M. Magruder

Veterans Affairs Medical Center

Follow this and additional works at: https://digitalcommons.unl.edu/publichealthresources

Part of the Public Health Commons

Grubaugh, Anouk L.; Long, Mary E.; Elhai, Jon D.; Frueh, B. Christopher; and Magruder, Kathryn M., "An examination of the construct validity of posttraumatic stress disorder with veterans using a revised criterion set" (2010). Public Health Resources. 175.

https://digitalcommons.unl.edu/publichealthresources/175

This Article is brought to you for free and open access by the Public Health Resources at DigitalCommons@University of Nebraska - Lincoln. It has been accepted for inclusion in Public Health Resources by an authorized administrator of DigitalCommons@University of Nebraska - Lincoln. 


\title{
An examination of the construct validity of posttraumatic stress disorder with veterans using a revised criterion set
}

\author{
Anouk L. Grubaugh ${ }^{\mathrm{a}, \mathrm{b}, *}$, Mary E. Long ${ }^{\mathrm{c}, 1}$, Jon D. Elhai ${ }^{\mathrm{d}}$, B. Christopher Frueh ${ }^{\mathrm{e}, 2}$, Kathryn M. Magruder ${ }^{\mathrm{a}, \mathrm{b}}$ \\ ${ }^{a}$ Ralph H. Johnson Veterans Affairs Medical Center, SC, USA \\ ${ }^{\mathrm{b}}$ Medical University of South Carolina, Behavioral, Epidemiologic, and Community Health Division, Department of Psychiatry and Behavioral Sciences, 67 President Street, \\ P.O. Box 250861, Charleston, SC 29425, USA \\ ${ }^{\mathrm{c}}$ Michael E. Debakey Veterans Affairs Medical Center and Baylor College of Medicine, Houston, TX, USA \\ ${ }^{\mathrm{d}}$ Department of Psychology, University of Toledo, Mail Stop \#948, 2801 West Bancroft Street, Toledo, OH 43606-3390, USA \\ e The Menninger Clinic and Baylor College of Medicine, Houston, TX, USA
}

\section{A R T I C L E I N F O}

\section{Article history:}

Received 16 July 2009

Received in revised form

24 March 2010

Accepted 20 May 2010

\section{Keywords:}

Posttraumatic stress disorder (PTSD)

DSM-IV

Construct validity

Veterans

Primary care

Factor analysis

\begin{abstract}
A B S T R A C T
Ongoing concerns exist in the literature regarding the construct of posttraumatic stress disorder (PTSD) and how to best conceptualize and measure this disorder. We compared the traditional DSM-IV PTSD symptom criteria (i.e., symptoms from clusters B, C, and D) to a revised criterion set that omits overlapping mood and other anxiety symptoms on PTSD prevalence, PTSD diagnostic caseness, associated psychiatric comorbidity, functional status, and structural validity using a cross-sectional, multi-site primary care sample of 747 veterans. After removing items theorized to overlap with mood and other anxiety disorders, PTSD prevalence was identical using both criterion sets (i.e., 12\%). Overall, there were few statistically significant differences in PTSD caseness, associated psychiatric comorbidity, functional status, and structural validity across the two diagnostic criterion sets. These data provide further support that removing items that overlap with other psychiatric disorders does not significantly impact the prevalence of PTSD, its associated comorbidity and functional impairment, or its structural validity. Although the revised criterion set represents a more parsimonious model, the current study findings generally support the strong construct validity of PTSD. The implications of these study findings for research and clinical practice are discussed.
\end{abstract}

Published by Elsevier Ltd.
Relative to other psychiatric diagnoses, posttraumatic stress disorder (PTSD) has undergone significant changes with regard to its conceptualization and symptom criteria inclusion since the addition of the disorder in the DSM-III (APA, 1980). There has even been some debate regarding the conceptual definition of criterion A, which serves as the index traumatic event used to assess the presence and severity of posttraumatic stress symptoms. These, and other, issues were recently highlighted in a special issue of The Journal of Anxiety Disorders (Rosen \& Frueh, 2007). Although not

\footnotetext{
* Correspondence to: Anouk L. Grubaugh, Medical University of South Carolina, Behavioral, Epidemiologic, and Community Health Division, Department of Psychiatry and Behavioral Sciences, 67 President Street, P.O. Box 250861, Charleston, SC 29425. Tel.: +1 843792 5215; fax: +1 8437926889 .

E-mail addresses: grubaugh@musc.edu (A.L. Grubaugh), mary.long5@va.gov (M E. Long), jonelhai@fastmail.fm (J.D. Elhai), frueh@hawaii.edu (B.C. Frueh), magrudkm@musc.edu (K.M. Magruder).

1 Houston Center for Quality of Care and Utilization Studies, Michael E. DeBakey VAMC, 2002 Holcombe Drive (152), Houston, TX 77030, USA.

2 University of Hawai'i at Hilo, Department of Psychology, 200 West Kawili Street Hilo, Hawai'i 96720, USA.
}

necessarily reflective of the PTSD community more broadly, the authors represented in this issue called for a more thorough examination of the critical issues and assumptions underlying the construct validity of PTSD and suggested ways to tighten the definition of trauma and improve the diagnostic specificity of PTSD (McHugh \& Treisman, 2007; Rosen \& Frueh, 2007; Spitzer, First, \& Wakefield, 2007).

In response to concerns regarding the construct validity of PTSD, Spitzer et al. (2007) recently proposed a revised criterion set that 1) includes a more stringent traumatic stressor criterion (criterion A) that excludes events that are not directly witnessed or experienced; 2 ) requires that symptoms develop within one week of the trauma; or in delayed cases, that the onset of symptoms be directly linked to a thematically related event; 3 ) ensures that symptoms are not due to an exacerbation of a pre-existing mood, anxiety, or personality disorder; and 4) eliminates acute, chronic, and delayed-onset specifiers. Additionally, and of relevance to our previous work and the current study, the diagnostic criterion set proposed by Spitzer and colleagues suggests removing five (5) symptoms from PTSD's criterion $\mathrm{C}$ and $\mathrm{D}$ clusters (i.e., the avoidance/numbing and 
hyperarousal clusters), four of which (C4-anhedonia, D1-sleep disturbance, D2-irritability, D3-impaired concentration) are theorized to overlap with mood and other anxiety disorders, and one of which (C3-impaired recall of the trauma) is difficult to disentangle from normal memory loss that can occur over time. Spitzer et al. (2007) further argue that the remaining criterion C and D symptoms should be combined into a single avoidance/hyperarousal cluster, requiring a minimum of 4 symptoms altogether for a diagnosis of PTSD.

Recent work compared the traditional DSM-IV criterion set to Spitzer et al.'s proposed refinement to the diagnosis using the National Comorbidity Survey Replication Data (NCS-R; Elhai, Grubaugh, Kashdan, \& Frueh, 2008). In that study, removing symptoms proposed to overlap with mood and other anxiety disorders modestly changed the lifetime prevalence of PTSD from $6.8 \%$ to $6.4 \%$. More specifically, approximately $13 \%$ of participants in the NCS-R no longer met criteria for PTSD using Spitzer et al.'s model and 7\% of individuals meeting Spitzer criteria for PTSD did not meet DSM-IV criteria for PTSD. Despite these differences, the level of agreement between both models was substantial (i.e., 98.3\% agreement). Additional findings indicated that implementing the symptom removals did not significantly alter diagnostic comorbidity and disability rates or significantly alter the construct validity of PTSD.

In separate analyses, the prevalence estimates, comorbidity rates, structural validity, and reliability of Spitzer et al.'s (2007) proposed symptom criterion set for the diagnosis of PTSD were compared to the DSM-IV criterion set in a representative community sample of adolescents (Ford, Elhai, Ruggiero, \& Frueh, 2009). PTSD prevalence varied across models (i.e., 5.2 to $8.2 \%$, lifetime, 3.2 to $5.0 \%$, past six months; for Spitzer et al.'s (2007) versus DSM-IV criterion sets, respectively). When a two-factor model was used with a proportionate symptom threshold, lifetime PTSD prevalence was comparable to that of the three-factor DSM-IV model, and lifetime major depressive episode lifetime comorbidity was reduced by $10-15 \%$. Comorbidity with substance use disorders was comparable across models. Structural validity, tested with confirmatory factor analyses, showed that Spitzer et al.'s two-factor model and a four-factor DSM-IV model were superior to the DSM-IV three-factor model.

The purpose of current paper was to replicate and extend previous examinations of Spitzer et al.'s (2007) proposed refinement to PTSD's criterion set, relative to the traditional DSM-IV PTSD criterion set, but applied to military veterans. Similar to previous work on this topic, we were specifically interested in examining PTSD prevalence, PTSD diagnostic caseness, psychiatric comorbidity, functional status, and structural validity across both diagnostic sets. This investigation is timely as efforts to revise the $D S M-V$ are currently in progress and there remains ongoing concern and discussion regarding how to improve the diagnostic accuracy of PTSD.

\section{Method}

\section{Study design}

A cross-sectional study was conducted on a random sample of veterans at four VA Medical Centers (Charleston and Columbia, SC; Tuscaloosa and Birmingham, AL). Study participants were randomly selected from a master list of patients during the fiscal year 1999 at one of the four VA primary care sites. Consenting participants were provided with a semi-structured clinic assessment at the time of informed consent, and within two months, were administered a structured telephone interview by masters level clinicians trained and supervised by a licensed clinical psychologist. This two-pronged approach was taken because the clinicians trained in the administration of the structured interview measures (i.e., CAPS and MINI; see below) were all located in one VA site in order to better monitor fidelity to the procedures and provide adequate supervision. Initial exclusionary criteria included the presence of dementia-related symptoms and being age 80 or older. After providing a complete description of the study to participants, written informed consent was obtained. This study was conducted with full approval from relevant Institutional Review Boards. See Magruder et al. (2005), addressing different research questions, for more study details.

\section{Participants}

A total of 1198 randomly identified veterans (known to be alive) were approached for study participation. Of this sample, 885 veterans (74\%) provided an informed consent to participate during the clinic interview. In this subset, 747 veterans were available for a telephone interview (84.4\%), 669 of which endorsed a trauma and were administered the Clinician Administered PTSD Scale (89.6\%).

\section{Clinic interview measures (face-to-face)}

Demographic Information. Participants were asked about sociodemographic and background information including age, gender, race, relationship status, education, living arrangement, and employment status.

The Short-Form Health Survey (SF-36; Ware \& Sherbourne, 1992) is a 36-item self-report measure of several health domains that yields two composite scores reflecting global physical and mental health functioning. The SF-36 has been shown to be a valid and reliable instrument for use with veteran populations (Richardson, Engel, Hunt, McKnight, \& McFall, 2002). For all scales, higher scores reflect greater health and functioning or functional status. In the present study, the two items comprising the Bodily Pain domain were not included in the physical health composite score because they were assessed using an incorrect response range. Despite this omission, however, our study findings yielded similar physical health composite scores relative to other veteran studies (Barrett et al., 2002; Dobie et al., 2004).

\section{Telephone interview measures}

Contact information of patients who completed the face-to-face clinic interview was sent to the Charleston VA Medical Center, where clinicians (master's level and above) telephoned them to administer a series of structured interviews, inclusive of the Trauma Assessment for Adults used to assess for Criterion A of PTSD, the Clinician Administered PTSD Scale, and the Mini International Neuropsychiatric Interview.

The Trauma Assessment for Adults-Self Report Version (Resnick, 1996) assesses the lifetime incidence of trauma (both military and non-military) and has been widely used to screen community and medical populations for trauma history (Gray, Elhai, Owen, \& Monroe, 2009; Kilpatrick et al., 2000). This survey provided data to categorize patients as either meeting or failing to meet DSM-IV PTSD criterion A.

The Clinician Administered PTSD Scale (CAPS; Blake et al., 1990) was administered to those participants who endorsed at least one trauma on the Trauma Assessment for Adults. The CAPS is a structured clinical interview that measures the intensity and frequency of the 17 DSM-IV PTSD symptoms (APA, 1994). The CAPS is considered the "gold standard" for assessing PTSD diagnoses with excellent psychometric properties and diagnostic utility (Weathers, Keane, \& Davidson, 2001). The CAPS was used to obtain current 
PTSD diagnoses according to the DSM-IV; and CAPS items were omitted and retained according to Spitzer et al. (2007) recommendations to yield revised current PTSD prevalence. Inter-rater reliability was calculated on $20 \%$ of cases using the DSM-IV criteria, and reviewers were $100 \%$ concordant on these diagnoses.

The DSM-IV requires at least one (1) symptom from the criterion B cluster, three (3) symptoms from the criterion C cluster, and two (2) symptoms from the criterion D cluster, for a total of at least six (6) required symptoms to yield a diagnosis of PTSD. The revised Spitzer model, on the other hand, requires at least one (1) symptom from the criterion B cluster and at least four (4) symptoms from the combined criterion C and D clusters, for a total of at least five (5) required symptoms for a diagnosis of PTSD. The scoring algorithm used the F1/12 rule, which is recommended by others (Weathers, Ruscio, \& Keane, 1999) as the optimal scoring rule for clinical and research purposes. For a symptom to be present and count toward a PTSD diagnosis, the frequency rating for that item had to be one or greater and the intensity rating had to be two or greater. The advantage of this scoring rule is that it serves as a diagnostic threshold allowing for the minimum number of symptoms for a diagnosis of PTSD.

The Mini International Neuropsychiatric Interview (MINI; Sheehan et al., 1998) is a brief structured interview that assesses the criteria for DSM-IV Axis I diagnoses (e.g., anxiety, mood, and substance abuse disorders; Lecrubier et al., 1997; Sheehan et al., 1998). The MINI exhibits similar sensitivity and specificity to more time-intensive structured psychiatric interviews (e.g., SCID) (Lecrubier et al., 1997; Sheehan et al., 1998). For the present study, the MINI was used to obtain current psychiatric diagnoses inclusive of major depression, generalized anxiety disorder (GAD), panic disorder, social phobia, and substance abuse/dependence.

\section{Chart review}

Trained chart reviewers examined electronic medical records for the 12 months preceding study initiation for each consenting patient. Research personnel who conducted chart reviews were blind to the diagnostic status of participants. Of relevance to this study, chart reviewers noted whether or not participant's served in a war zone and whether or not they were receiving disability compensation through the VA.

\section{Sample description}

Univariate descriptive statistics and frequency distributions were obtained for the demographic variables, as well as PTSD prevalence, associated psychiatric comorbidity, disability, and functional status.

\section{Primary analyses}

All analyses were two-tailed and conducted using SPSS 16 for Windows, except when examining differences in PTSD structural validity, for which Mplus 5.2 (Muthén \& Muthén, 1998-2007) was used. Continuous variables were screened for missing values and outliers prior to conducting the primary analyses.

The binomial approximation $z$ test statistic for proportions was used to investigate whether Spitzer et al.s' (2007) symptom deletions resulted in statistically significant differences in PTSD prevalence, PTSD diagnostic status, associated psychiatric comorbidity, and disability. $T$-tests were used to investigate whether Spitzer et al. s' (2007) symptom deletions resulted in statistically significant differences in physical and mental health functioning scores as measured by the SF-36. Confirmatory factory analyses (CFAs) were conducted in order to examine the impact of symptom deletions on the structural validity of PTSD.

\section{Results}

Demographic

Sample demographics and trauma exposure variables are presented in Table 1.

\section{Prevalence}

Ninety (90) patients (12.0\%) met criteria for PTSD using both the DSM-IV PTSD criteria and Spitzer et al.'s (2007) proposed symptom criteria (see Table 1). No significant difference was found between the two PTSD symptom structure models in terms of diagnostic status. In fact, only 4 participants (.5\%) who met PTSD criteria according to DSM-IV did not receive a PTSD diagnosis when using Spitzer and colleagues' revised symptom criterion set, and only 4 participants (.5\%) lost their current PTSD diagnosis when going

\section{Table 1}

PTSD prevalence and sample demographics.

\begin{tabular}{|c|c|}
\hline Categorical variables & $\begin{array}{l}\text { Current PTSD sample } \\
(n=747) \\
n(\%)\end{array}$ \\
\hline DSM-IV PTSD Criteria & $90(12.0)$ \\
\hline Spitzer PTSD Criteria & $90(12.0)$ \\
\hline \multicolumn{2}{|l|}{ Gender } \\
\hline Male & $696(93.2)$ \\
\hline \multicolumn{2}{|l|}{ Race } \\
\hline Caucasian & $468(62.7)$ \\
\hline African-American & 259 (34.7) \\
\hline All Other ${ }^{\mathrm{a}}$ & $6(.8)$ \\
\hline \multicolumn{2}{|l|}{ Relationship Status } \\
\hline Living Alone & $221(29.6)$ \\
\hline Living with Someone & $525(70.3)$ \\
\hline \multicolumn{2}{|l|}{ Work Status } \\
\hline Working & 246 (32.9) \\
\hline Not Working (Retired) & $281(37.6)$ \\
\hline Not Working (Disabled) & $220(29.5)$ \\
\hline \multicolumn{2}{|l|}{ Education } \\
\hline$\leq$ High School & $379(50.7)$ \\
\hline Some College & $249(33.3)$ \\
\hline College/Post Graduate & $119(15.9)$ \\
\hline \multicolumn{2}{|l|}{ VA Study Site } \\
\hline Charleston & $220(29.5)$ \\
\hline Columbia & 238 (31.9) \\
\hline Tuscaloosa & $177(23.7)$ \\
\hline Birmingham & $112(15.0)$ \\
\hline \multicolumn{2}{|l|}{ Criterion A Event $^{\mathrm{b}}$} \\
\hline Combat Exposure & $370(50.2)$ \\
\hline Physical Assault & $173(23.6)$ \\
\hline Adult Sexual Assault & $17(2.5)$ \\
\hline Child Sexual Assault & $44(6.3)$ \\
\hline Serious Accident & $265(36.1)$ \\
\hline Continuous Variables & $M(S D)$ \\
\hline Age & $61.2(11.8)$ \\
\hline \multicolumn{2}{|l|}{ CAPS $^{c}$} \\
\hline DSM-IV Criteria & $13.02(26.3)$ \\
\hline Spitzer et al. Criteria & $9.96(20.4)$ \\
\hline
\end{tabular}


from Spitzer et al.'s criterion set to the DSM-IV PTSD criteria. In total, only $1.0 \%$ of the entire sample ( 8 out of 747 participants) changed diagnostic status for current PTSD when using Spitzer et al.'s symptom deletions, with substantial level of agreement between the two diagnostic models (94.3\% agreement, Kappa $=.94$, $p<.001)$.

\section{Psychiatric comorbidity, health functioning, and disability}

Next we examined if the PTSD symptom structure models were associated with different rates of comorbid psychiatric disorders and other health indicators. Results indicated that changes in PTSD symptom criteria did not produce statistically significant differences in psychiatric comorbidity, mental and physical health functioning, or disability rates (see Table 2 ).

\section{Structural validity}

We used Mplus 5.2 software (Muthén \& Muthén, 1998-2007) to examine the impact of symptom deletions on PTSD's structural validity (see Table 3 ). Analyses were two-tailed, and no missing values were present (given the nature of structured diagnostic interviewing), thus yielding a sample size of 669 participants endorsing trauma exposure.

We conducted confirmatory factor analyses (CFAs) for the PTSD diagnostic systems. We first tested how well Spitzer et al.'s (2007) covariance matrices for the two-factor PTSD model (re-experiencing and avoidance/hyperarousal; implementing the five-symptom deletion) related to the 12 corresponding CAPS PTSD symptoms, with factors allowed to covary and error covariances fixed to zero. We compared this model's results to the standard three-factor (17item) DSM-IV PTSD model (reexperiencing, avoidance/numbing, hyperarousal). However, given empirical evidence for separating PTSD criterion C's effortful avoidance and emotional numbing into two separate factors (Asmundson, Stapleton, \& Taylor, 2004), we also tested such a four-factor structure.

\section{Table 2}

Differences in diagnostic comorbidity, disability, and functional status between DSM-IV and Spitzer et al.'s (2007) PTSD criterion set.

\begin{tabular}{|c|c|c|c|}
\hline \multirow[t]{2}{*}{ Comorbid with PTSD } & \multicolumn{3}{|l|}{ Current PTSD } \\
\hline & $\begin{array}{l}\text { DSM-IV PTSD } \\
n=90 \\
n(\%)\end{array}$ & $\begin{array}{l}\text { Spitzer et al. PTSD } \\
n=90 \\
n(\%)\end{array}$ & $Z^{\mathrm{b}}$ \\
\hline \multicolumn{4}{|l|}{ Specific Disorders $^{\mathrm{a}}$} \\
\hline MDD & $62(68.1)$ & $61(67.8)$ & .06 \\
\hline GAD & $38(41.8)$ & $36(40.0)$ & .34 \\
\hline Panic & $36(39.6)$ & $36(40.0)$ & .08 \\
\hline Social phobia & $19(20.9)$ & $18(20.0)$ & .21 \\
\hline Substance & $10(11.0)$ & $11(12.2)$ & .36 \\
\hline \multicolumn{4}{|l|}{ Across disorders } \\
\hline Only PTSD & $13(14.4)$ & $15(16.7)$ & .62 \\
\hline 1 diagnosis & $21(22.1)$ & $20(22.2)$ & .02 \\
\hline 2 diagnoses & $31(34.1)$ & $30(33.3)$ & .16 \\
\hline$\geq 3$ diagnoses & $25(27.5)$ & $25(27.8)$ & .06 \\
\hline \multirow[t]{2}{*}{ Disability } & $43(47.8)$ & $44(48.9)$ & .21 \\
\hline & $m(s d)$ & $m(s d)$ & $t^{c}$ \\
\hline Physical Health Functioning & $39.43(19.25)$ & $39.00(19.31)$ & .14 \\
\hline Mental Health Functioning & $37.89(21.23)$ & $37.38(21.02)$ & .15 \\
\hline \multicolumn{4}{|l|}{$\begin{array}{l}p<.05 \\
\text { a } M D D=\text { major depressive }\end{array}$} \\
\hline \multicolumn{4}{|c|}{ Substance = substance abuse/dependence. } \\
\hline
\end{tabular}

Variable distributions for current PTSD symptom ratings were substantially skewed and kurtotic based on multivariate and univariate tests using DSM-IV and Spitzer criteria; consequently, CFAs used maximum likelihood estimation with a mean-adjusted Satorra-Bentler (S-B) chi-square statistic (Satorra \& Bentler, 2001). Chi-square tests of model fit were examined in conjunction with relative and absolute goodness-of-fit indices, including the Tucker-Lewis index (TLI), comparative fit index (CFI), root mean square error of approximation (RMSEA), and standardized root mean square residual (SRMR) [interpreted as excellent fit when RMSEA $\leq .06$, SRMR $\leq .08$, CFI/TLI $>.95$ ] (Hu \& Bentler, 1998, 1999).

Spitzer et al.'s (2007) two-factor model (having removed overlapping mood and anxiety symptoms) provided an adequate fit, $\mathrm{S}-\mathrm{B} \chi^{2}(53, N=669)=274.65, p<.001$, TLI $=.91, \mathrm{CFI}=.93$, RMSEA $=.08$, SRMR $=.03$. A similar 12 -item model that separated the avoidance and hyperarousal factors also provided an adequate fit, S-B $\chi^{2}(51, N=309)=243.02, p<.001$, TLI $=.92$, CFI $=.94$, $\mathrm{RMSEA}=.08, \mathrm{SRMR}=.03$, and represented a small but statistically significant improvement over the two-factor model, $\mathrm{S}-\mathrm{B} \chi^{2}$ change $(2$, $N=669)=27.84, p<.001$. The three-factor DSM-IV PTSD model yielded an adequate fit, $\mathrm{S}-\mathrm{B} \chi^{2}(116, N=669)=330.02, p<.001$, $\mathrm{TLI}=.94, \mathrm{CFI}=.95, \mathrm{RMSEA}=.05, \mathrm{SRMR}=.03$. Fit indices were only slightly better than Spitzer et al.'s (2007) model with three-factors. When separating the three-factor DSM-IV model's avoidance and numbing symptoms to derive a four-factor model, an excellent fit resulted, $\mathrm{S}-\mathrm{B} \chi^{2}(113, N=669)=184.96, p<.001$, TLI $=.98$, $\mathrm{CFI}=.98, \mathrm{RMSEA}=.03, \mathrm{SRMR}=.02$, representing a significant improvement over the three-factor model, S-B $\chi^{2}$ change(3, $N=669)=125.29, p<.001$.Thus, a 4 -factor model with 17 items provided a superior fit for the data.

\section{Discussion}

In light of ongoing discussion regarding the construct validity of PTSD, the current manuscript examined Spitzer et al.'s (2007) proposed refinement to PTSD's criterion set, relative to the traditional DSM-IV PTSD criterion set, in a sample of veterans. We were specifically interested in examining prevalence of PTSD, PTSD diagnostic caseness, psychiatric comorbidity, functional status, and structural validity across both diagnostic sets. After removing items theorized to overlap with mood and other anxiety disorders, current prevalence of PTSD remained unaltered (i.e., 12.0\%) using $D S M-I V$ and Spitzer's criteria. Four participants (.5\%) who met criteria for PTSD according to the DSM-IV no longer met criteria using the Spitzer et al. (2007) model, and conversely, only four participants (.5\%) lost their diagnosis going from the Spitzer et al. diagnostic system to the DSM-IV diagnostic system.

Worth noting, there is a substantial level of agreement between the two diagnostic models (94.3\% agreement, Kappa $=.94$, $p<.001)$. The concordance in PTSD prevalence between the two diagnostic sets is remarkably similar, even more so than previous findings using a representative adult community sample (Elhai et al., 2008). However, these findings differ from similar analyses using a nationally representative community sample of adolescents, which yielded statistically significant differences in PTSD prevalence between the two diagnostic sets [i.e., the Spitzer et al. (2007) model lead to lower prevalence estimates than the DSM$I V$; Ford et al., 2009]. However, when Ford et al. (2009) compared the DSM-IV to a revised Spitzer et al. (2007) model with a relaxed three versus four C/D symptom threshold, statistically significant differences in PTSD prevalence disappeared between the two diagnostic models. Altogether, these findings suggest that removing items theorized to overlap with mood and anxiety disorders do not significantly impact PTSD diagnostic rates or caseness. 
Table 3

Confirmatory factor-analytic models examined.

\begin{tabular}{|c|c|c|c|c|}
\hline DSM-IV PTSD Items & Spitzer et al., 20072 factors & Spitzer et al., 20073 factors & DSM-IV, 3 factors & DSM-IV, 4 factors \\
\hline B1. Intrusive thoughts & Reexperiencing & Reexperiencing & Reexperiencing & Reexperiencing \\
\hline B2. Nightmares & Reexperiencing & Reexperiencing & Reexperiencing & Reexperiencing \\
\hline B3. Flashbacks & Reexperiencing & Reexperiencing & Reexperiencing & Reexperiencing \\
\hline B4. Cued emotional reactivity & Reexperiencing & Reexperiencing & Reexperiencing & Reexperiencing \\
\hline B5. Cued physical reactivity & Reexperiencing & Reexperiencing & Reexperiencing & Reexperiencing \\
\hline C1. Avoiding thoughts & Avoidance/Hyperarousal & Avoidance & Avoidance/Numbing & Avoidance \\
\hline C2. Avoiding triggers/reminders & Avoidance/Hyperarousal & Avoidance & Avoidance/Numbing & Avoidance \\
\hline C3. Specific amnesia & NA & NA & Avoidance/Numbing & Numbing \\
\hline C4. Loss of interest & NA & NA & Avoidance/Numbing & Numbing \\
\hline C5. Feeling distant & Avoidance/Hyperarousal & Avoidance & Avoidance/Numbing & Numbing \\
\hline C6. Feeling numb & Avoidance/Hyperarousal & Avoidance & Avoidance/Numbing & Numbing \\
\hline C7. Foreshortened sense of future & Avoidance/Hyperarousal & Avoidance & Avoidance/Numbing & Numbing \\
\hline D1. Sleep disturbance & NA & NA & Hyperarousal & Hyperarousal \\
\hline D2. Irritability & NA & NA & Hyperarousal & Hyperarousal \\
\hline D3. Difficulty concentrating & NA & NA & Hyperarousal & Hyperarousal \\
\hline D4. Hypervigilance & Avoidance/Hyperarousal & Hyperarousal & Hyperarousal & Hyperarousal \\
\hline D5. Exaggerated startle response & Avoidance/Hyperarousal & Hyperarousal & Hyperarousal & Hyperarousal \\
\hline
\end{tabular}

$\mathrm{NA}=$ not applicable-item removed by Spitzer et al., 2007.

In the current sample, there were no statistically significant differences in associated psychiatric comorbidity, functional status, and disability across the two diagnostic criterion sets. These findings are also similar to previous studies comparing Spitzer et al.'s proposed criteria to the DSM-IV (Elhai et al., 2008; Ford et al., 2009). With regard to the structural validity of PTSD, Spitzer et al.'s (2007) proposed criterion set and the DSM-IV 3-factor model provided an adequate fit for the data. These findings are again similar to the previous study using an adult community sample (Elhai et al., 2008), but they differ somewhat from the previous study using a community sample of adolescents (Ford et al., 2009). This latter study found that Spitzer et al.'s proposed criterion set provided a superior fit to the data relative to the DSM-IV 3-factor analytic model, albeit with adolescents rather than adults. However, worth noting, all three studies supported a 4 -factor, 17 -item model as providing a superior fit for the data; and these findings are consistent with other studies on this topic (Asmundson et al., 2004; Simms, Watson, \& Doebbeling, 2002).

It should be noted as a study limitation that we did not test all of Spitzer et al.'s (2007) recommended changes to the DSM-IV definition of PTSD, including important suggested changes regarding the traumatic stressor criterion (criterion A). However, largely due to the nature of our sample, all PTSD positive individuals directly witnessed or experienced at least one criterion A event. Additionally, there were few cases of delayed-onset PTSD after 1 month of exposure (Frueh, Grubaugh, Yeager, \& Magruder, 2009). Only including criterion A events that were directly witnessed or experienced and ensuring that cases of delayed onset can be directly linked to a thematically related event are some of Spitzer et al.'s most notable recommended changes to the traumatic stressor criterion. In this regard, it is unlikely that inclusion of these additional recommended changes would have yielded significantly different findings. Also worth noting, the current sample was drawn from four primary care clinics and PTSD cases in primary care may be less severe than those found in mental health specialty care and include a higher percentage of cases at the threshold for diagnosis. A higher number of such cases could have altered the study findings. Finally, and on a related note, we chose to use the preferred and most common scoring rule for determining a PTSD diagnosis in order to generalize our results to the broader PTSD literature. Other scoring methods, however, could have yielded different results than those obtained in the current study.

Altogether, when applying Spitzer et al.'s (2007) criterion set to adult samples, there are few statistically significant differences in
PTSD prevalence, psychiatric comorbidity, functional status, and disability relative to DSM-IV's criterion set. Thus, these data support previous findings that removing symptoms theorized to overlap with other anxiety and mood disorders does not appear to significantly change PTSD prevalence or diagnostic caseness at the patient level. This interpretation hints at some important practical and conceptual implications. On a practical level, it suggests that PTSD diagnoses can be reliably made with fewer items (i.e., using a more parsimonious symptom criterion set). Additionally, the items that could be removed without significant loss to the diagnostic accuracy of PTSD are those items which are often difficult to disentangle from the symptoms of other psychiatric disorders in clinical practice settings (i.e., loss of interest in activities related to PTSD versus loss of interest due to depression). Thus, screening and assessment measures could be significantly shortened and simplified for both research and clinical purposes. However, on a conceptual level, the current data speaks to the strength of the construct validity of PTSD. As development of the DSM-V is in progress, it is important for the field to better understand and improve the diagnostic accuracy and construct validity of PTSD, including the accuracy of its factor structure and parsimony with other diagnostic constructs. Such efforts should include the examination of additional symptoms and criteria that would enhance PTSD case identification, rather than focus exclusively on symptoms that should be deleted.

\section{Acknowledgement}

This work was partially supported by grants CD207015 and VCR99-010-2 from the Veterans Affairs Health Services Research and Development (HSR\&D) program and grant MH074468 from the National Institute of Mental Health (NIMH).

\section{References}

American Psychiatric Association. (1980). Diagnostic and statistical manual of mental disorders (3rd ed.). Washington, DC: American Psychiatric Press.

American Psychiatric Association. (1994). Diagnostic and statistical manual of mental disorders (3rd ed.). Washington, DC: American Psychiatric Press.

Asmundson, G. J. G., Stapleton, J. A., \& Taylor, S. (2004). Are avoidance and numbing distinct PTSD symptom clusters? Journal of Traumatic Stress, 17, 467-475.

Barrett, D. H., Doebbeling, C. C., Schwartz, D. A., Voelker, M. D., Falter, K. H., Woolson, R. F., et al. (2002). Posttraumatic stress disorder and self-reported physical health status among U.S. military personnel serving during the Gulf War period. Psychosomatics, 43, 195-205.

Blake, D. D., Weathers, F. W., Nagy, L. N., Kaloupek, D. G., Klauminzer, G., Charney, D. S., et al. (1990). A clinician rating scale for assessing current and lifetime PTSD: the CAPS-1. The Behavior Therapist, 18, 187-188. 
Dobie, D. J., Kivlahan, D. R., Maynard, C., Bush, K. R., Davis, T. M., \& Bradley, K. A. (2004). Posttraumatic stress disorder in female veterans: association with selfreported health problems and functional impairment. Archives of Internal Medicine, 164, 394-400

Elhai, J. D., Grubaugh, A. L., Kashdan, T. B., \& Frueh, B. C. (2008). Empirical examination of a proposed refinement to DSM-IV posttraumatic stress disorder symptoms criteria using the National comorbidity survey replication data. Journal of Clinical Psychiatry, 69, 597-602.

Ford, J. D., Elhai, J. D., Ruggiero, K. J., \& Frueh, B. C. (2009). Refining posttraumatic stress disorder diagnosis: evaluation of symptom criteria with the National survey of adolescents. Journal of Clinical Psychiatry, 70, 748-755.

Frueh, B. C., Grubaugh, A. L., Yeager, D. E., \& Magruder, K. M. (2009). Delayed-onset post-traumatic stress disorder among war veterans in primary care. British Journal of Psychiatry, 194, 515-520.

Gray, M. J., Elhai, J. D., Owen, J. R., \& Monroe, J. R. (2009). Psychometric properties of the trauma assessment for adults. Depression and Anxiety, 26, 190-195.

Hu, L., \& Bentler, P. M.(1998). Fit indices in covariance structural modeling: sensitivity to underparameterized model misspecification. Psychological Methods, 3, 424-453.

Hu, L., \& Bentler, P. M. (1999). Cutoff criteria for fit indexes in covariance structure analysis: conventional criteria versus new alternatives. Structural Equation Modeling, 6, 1-55.

Kilpatrick, D. G., Acierno, R., Saunders, B., Resnick, H. S., Best, C. L., \& Schnurr, P. P. (2000). Risk factors for adolescent substance abuse and dependence: data from a national sample. Journal of Consulting and Clinical Psychology, 68, 19-30.

Lecrubier, Y., Sheehan, D. V., Weiller, E., Amorim, P., Bonora, I., Sheehan, K. H., et al. (1997). The Mini International Neuropsychiatric Interview (MINI). A short diagnostic structured interview: reliability and validity according to the CIDI. European Psychiatry, 12, 224-231.

McHugh, P. R., \& Treisman, G. (2007). PTSD: a problematic diagnostic category. Journal of Anxiety Disorders, 21, 211-222.

Magruder, K. M., Frueh, B. C., Knapp, R. G., Davis, L., Hamner, M. B., Martin, R. H., et al. (2005). Prevalence of posttraumatic stress disorder in VA primary care clinics. General Hospital Psychiatry, 27, 169-179.
Muthén, L. K., \& Muthén, B. O. (1998-2007). Mplus user's guide (5th ed.). Los Angeles, California: Muthén \& Muthén.

Resnick, H. S. (1996). Psychometric review of Trauma Assessment for Adults (TAA) In B. H. Stamm (Ed.), Measurement of stress, trauma, and adaptation. Lutherville, MD: Sidran Press.

Richardson, R. D., Engel, C. C., Jr., Hunt, S. C., McKnight, K., \& McFall, M. (2002). Are veterans seeking Veterans affairs' primary care as healthy as those seeking department of defense primary care? A look at gulf war veterans' symptoms and functional status. Psychosomatic Medicine, 64, 676-683.

Rosen, G. M., \& Frueh, B. C. (2007). Challenges to the PTSD construct and its database: the importance of scientific debate. Journal of Anxiety Disorders, 21 161-163.

Satorra, A., \& Bentler, P. M. (2001). A scaled difference chi-square test statistic for moment structure analysis. Psychometrika, 66, 507-514.

Sheehan, D. V., Lecrubier, Y., Sheehan, K. H., Amorim, P., Janavs, J., Weiller, E. et al. (1998). The Mini-International Neuropsychiatric Interview (MINI): the development and validation of a structured diagnostic psychiatric interview for DSM-IV and ICD-10. Journal of Clinical Psychiatry, 59(Suppl. 20), $22-33$.

Simms, L. J., Watson, D., \& Doebbeling, B. N. (2002). Confirmatory factor analyses of posttraumatic stress symptoms in deployed and nondeployed veterans of the Gulf War. Journal of Abnormal Psychology, 111, 637-647.

Spitzer, R. L., First, M. B., \& Wakefield, J. C. (2007). Saving PTSD from itself in DSM-V. Journal of Anxiety Disorders, 21, 233-241.

Ware, J. E., \& Sherbourne, C. D. (1992). The MOS 36-item short form health survey (SF-36). I. Conceptual framework and item selection. Medical Care, 30 473-483.

Weathers, F. W., Keane, T. M., \& Davidson, J. R. T. (2001). Clinician administered PTSD scale: a review of the first ten years of research. Depression and Anxiety, 13, 132-156.

Weathers, F. W., Ruscio, A. M., \& Keane, T. M. (1999). Psychometric properties of nine scoring rules for the clinician-administered posttraumatic stress disorder scale. Psychological Assessment, 11, 124-133. 